\title{
Cultivo de girassol ornamental (Helianthus annuus L.) em vaso sob diferentes doses de paclobutrazol ${ }^{(1)}$
}

\author{
JOSÉ GERALDO BARBOSA(2), MAURICIO SOARES BARBOSA(3), SUSAN SATIE TSUJ((4), MOISÉS ALVES MUNIZ(3), JOSÉ \\ ANTÔNIO SARAIVA GROSSI(2) e MARIANA RUBIM(4)
}

\begin{abstract}
RESUMO
O girassol apresenta facilidade de propagação - tempo curto para colheita, e uma inflorescência atrativa, o que aumenta sua procura para a confecção de arranjos e vasos. $\mathrm{O}$ grande crescimento natural tem sido um dos entraves para que $\mathrm{o}$ girassol seja explorado como planta ornamental. O paclobutrazol é um regulador de crescimento que inibe a biossíntese de giberelina e reduz o alongamento da haste, podendo ser usado em diversas espécies ornamentais. Assim, para verificar a resposta de plantas de girassol, variedade Golden, dobrado, cultivado em vaso com diferentes substratos à aplicação de paclobutrazol, instalou-se experimento, utilizando-se o delineamento experimental em blocos casualizados em arranjo fatorial 4 × 4 com quatro substratos (solo:areia:casca de arroz carbonizada 2:0,5:2 v/v; solo:areia:carvão de cana, 2:0,5:2 $\mathrm{v} / \mathrm{v}$; solo:areia:carvão vegetal 2:0,5:2 v/v e substrato comercial) e quatro doses de paclobutrazol $(0 ; 2 ; 4 ; 6 \mathrm{mg}$ i.a./vaso) com 3 repetições. Avaliaram-se os parâmetros altura de planta $(\mathrm{cm})$, número de folhas, concentração de clorofila em unidades SPAD. Verificou-se melhor qualidade nas plantas cultivadas nos substratos que continham casca de arroz carbonizada e carvão de cana. Houve aumento da unidade SPAD com o aumento das doses de paclobutrazol, sendo o máximo estimado na dose de 5,08 mg i.a./vaso. Observou-se uma relação inversa entre a dosagem de paclobutrazol e a altura da planta, o que permite constatar que a aplicação da dose de $6 \mathrm{mg}$ i.a./vaso reduziu o porte das plantas de girassol, possibilitando boa harmonia de vaso, sendo, portanto, a mais recomendada.
\end{abstract}

Palavras-chave: girassol, planta envasada, paclobutrazol.

\section{ABSTRACTS}

Cultivation of ornamental sunflower (Helianthus annuus L.) in vase under different paclobutrazol doses

\begin{abstract}
The sunflower presents propagation easiness, short time for crop and an inflorescence attractive and suitable for arrangements and vases. The great natural growth has been one of the impediments for the sunflower to be explored as ornamental plant. The paclobutrazol, a growth regulator that inhibits the biosynthesis of gibberellins, could be used in several ornamental species, reducing the prolongation of stems. An experiment was conducted to investigate the effect of paclobutrazol on potted 'Golden' Sunflower. An 4x4 factorial block randomized designed experiment was established with 4 substrates (soil:sand:carbonized rice husk 2:0,5:2 v/v; soil:sand:fiber sugar cane coal 2:0,5:2 v/v; soil:sand:woodcoal 2:0,5:2 v/v; commercial potting media) and 4 paclobutrazol dosages $(0 ; 2 ; 4$ and $6 \mathrm{mg}$ a.i/pot) with 3 replications. The following characteristics were evaluated: plant height, number of leaves and chlorophyll content based on spad units. Plants grown on substrates with carbonized rice husk and woodcoal exhibited better quality. There was increase of the SPAD units with the increase of the paclobutrazol dosages, and its maximum value was noticed in the dose of 5,08 $\mathrm{mg}$ a.i./pot. Paclobutrazol dosages exhibited inverse relation to plant height. The dosage $6 \mathrm{mg}$ a.i./pot of paclobutrazol can be recommended to provide good pot plant harmony.
\end{abstract}

Key words: sunflower, potted plant, paclobutrazol.

\section{INTRODUÇÃO}

O girassol tem sido cultivado principalmente como planta industrial para a obtenção de óleo. Nos últimos anos, o surgimento de variedades com alto potencial ornamental tem possibilitado sua produção como flor de corte, sendo também cultivado como planta ornamental em vaso, embora, para isso, tenha que se utilizar variedades de porte baixo. Mesmo com porte reduzido, para obtenção de uma boa harmonia de vaso, faz-se necessário monitorar seu crescimento.

A utilização de retardantes de crescimento para a obtenção de plantas compactas tem sido pesquisada em espécies como crisântemo, hortênsias e outras, obtendose plantas de menor tamanho e sem deformações (WEAVER, 1989). Inibidores de crescimento em culturas

\footnotetext{
(1) Recebido para publicação em 04/07/2007 e aceito em 04/08/2008

(2) Departamento de Fitotecnia, Universidade Federal de Viçosa - UFV,, 36570-000 Viçosa (MG). E-mail.jgeraldo@ufv.br

(3) Doutorando em Fitotecnia, UFV.

(4) Estudante de Agronomia, UFV.
} 
hortícolas são cada vez mais avaliados em trabalhos de pesquisa, objetivando, sobretudo, a precocidade e o aumento da produtividade. Os produtos pertencentes ao grupo dos inibidores da biossíntese da giberelina, [CCC (Cloreto de 2-cloroetil-trimetil amônio), paclobutrazol e uniconazole], em geral têm sido utilizados em várias espécies (NORCINI et al., 1996).

MAUK et al. (1990) observaram que a inibição de crescimento vegetativo associada ao paclobutrazol tem sido ligada ao método de aplicação. Assim, ALMEIDA e PEREIRA (1996), aplicando paclobutrazol $\left(10^{-3} \mathrm{~mol}^{\left.-L^{-1}\right)}\right.$ em plantas de girassol cultivar 33 (Fundação Cargil), na parte aérea ou no solo, observaram que apenas a aplicação no solo causou nanismo nas plantas e atrasou o desenvolvimento dos primórdios foliares. Os resultados caracterizaram o forte envolvimento de substâncias giberelínicas no desenvolvimento vegetativo das plantas.

SETIA et al. (1995), de forma semelhante, observaram redução em altura de plantas de Brassica carinata, poucos dias após o tratamento com paclobutrazol. A aplicação via solo é mais efetiva para controlar o crescimento, pois o regulador é rapidamente absorvido pelas raízes e rapidamente translocado para a parte aérea, mas de acordo com HUNTER e PROCTOR (1992) e PENG et al. (1994), apenas uma pequena fração de ingrediente ativo chega aos meristemas apicais, onde ocorrem os efeitos inibitórios.

O girassol pode ser cultivado em diversos substratos, sendo determinantes na absorção do regulador pelas raízes, pois a capacidade de o substrato adsorver o regulador define a disponibilidade deste para as raízes. Quanto maior a adsorção do regulador pelo substrato, menor lixiviação e maior disponibilidade para a absorção do regulador.

O objetivo deste trabalho foi avaliar a resposta de plantas de girassol, cultivadas em vaso, com diferentes substratos, à aplicação de doses de paclobutrazol e verificar a dose mais eficiente na redução do porte da planta, de acordo com as exigências do mercado.

\section{MATERIAL E MÉTODOS}

O experimento foi desenvolvido no Setor de Floricultura, do Departamento de Fitotecnia da Universidade Federal de Viçosa (UFV), sob condições de casa de vegetação. As mudas de girassol da variedade Golden foram transplantadas no dia 16/09/06 e cultivadas em vaso 13 (volume $=0,8 \mathrm{dm}^{3}$, altura $=12 \mathrm{~cm}$ ), utilizandose uma muda por vaso. A fertirrigação foi realizada de 2 em 2 dias, aplicando-se volume de $150 \mathrm{~mL}$ da solução nutritiva recomendada por BARBOSA (2003): 11,51; 2,88; 1,$95 ; 12,92 ; 2,5 ; 1,0 ; 2,44$ mmol.L $\mathrm{L}^{-1}$ de N-NO${ }_{3}^{-}, \mathrm{N}^{-} \mathrm{NH}_{4}^{+}$, P- $\mathrm{H}_{2} \mathrm{PO}_{4}^{-}, \mathrm{K}^{+}, \mathrm{Ca}^{2+}, \mathrm{Mg}^{2+}, \mathrm{S}-\mathrm{SO}_{4}^{-2}$ e $30 ; 5 ; 50 ; 40 ; 0,1 ; 2$ ìmol. $\mathrm{L}^{-1} \mathrm{de} \mathrm{B}, \mathrm{Cu}, \mathrm{Fe}, \mathrm{Mn}, \mathrm{Mo}, \mathrm{Zn}$, respectivamente.

O paclobutrazol foi aplicado diretamente no substrato aos 7 e 21 dias após o plantio, sendo administrada metade da dose em cada período.

Avaliaram-se os parâmetros altura de planta $(\mathrm{cm})$, número de folhas e concentração de clorofila em unidades SPAD. A medida indireta de clorofila foi obtida com o auxílio de um medidor portátil de clorofila, quantificada em unidades SPAD.

Utilizou-se o delineamento experimental em blocos casualizados em esquema fatorial 4 x 4 com quatro tipos de substratos: S1: solo:areia:casca de arroz carbonizada $(2: 0,5: 2 \mathrm{v} / \mathrm{v}) ; \mathrm{S} 2$ : solo:areia:carvão de cana $(2: 0,5: 2 \mathrm{v} / \mathrm{v}) ; \mathrm{S} 3:$ solo:areia:carvão vegetal $(2: 0,5: 2 \mathrm{v} /$ v); S4: substrato comercial (Bioplant) e quatro doses de paclobutrazol $(0 ; 2 ; 4 ; 6 \mathrm{mg}$ de ingrediente ativo/vasoi.a./vaso) com 3 repetições - um vaso por repetição. No preparo das misturas aplicou-se um grama de adubo NPK 0-10-10 por Litro.

Os dados obtidos foram submetidos à análise da variância e de regressão, utilizando-se as rotinas do SAS/ STAT, sendo as médias testadas pelo teste de Tukey a $5 \%$ de probabilidade, para os tratamentos qualitativos. Para os tratamentos quantitativos foram ajustados modelos de regressão, selecionando-se dentro das equações de regressão, aquelas de efeito significativo, pelo teste $\mathrm{F}$, a 1 e $5 \%$ de probabilidade e de maior $\mathrm{R}^{2}$.

\section{RESULTADOS E DISCUSSÃO}

Quanto à altura da planta, a análise da variância mostrou efeito significativo para substrato, dose de paclobutrazol e para a interação a 5\% de probabilidade. $\mathrm{Na}$ ausência do paclobutrazol, os substratos S1 e S2 mostraram-se mais eficientes em reduzir a altura das plantas. O mesmo ocorreu quando se aplicou o regulador; maior eficiência foi apresentada com a dose de $6 \mathrm{mg}$ i.a./vaso. O S2 proporcionou as menores alturas de plantas em todas as doses aplicadas, demonstrando ser o mais recomendado para a aplicação do regulador, enquanto o S3 proporcionou a maior altura de plantas em todas as doses aplicadas, o que indica a redução da eficiência do paclobutrazol e, provavelmente, que o regulador foi pouco adsorvido pelo substrato, ficando menos disponível para ser absorvido pelas raízes das plantas (figura 1).

De acordo com a figura 1, observa-se efeito linear e inversamente proporcional entre a dose de paclobutrazol e a altura de planta. Este resultado demonstra o efeito do regulador sobre o crescimento das plantas de girassol. Houve uma redução média de $14,76 \%, 21,33 \%$ e $32,32 \%$ na altura das plantas quando se aplicou paclobutrazol nas doses de 2, 4 e $6 \mathrm{mg}$ i.a./ vaso respectivamente. DASOJU et al. (1998), trabalhando com girassol var. Pacino, observaram resultados semelhantes, cuja aplicação do produto em substrato (solo:esfagno: perlita, 2:5:5, v/v) causou redução de altura até uma dose de $16 \mathrm{mg}$ i.a/vaso. TINOCO et al. (2004), estudando o efeito de doses de paclobutrazol em crisântemos de vaso, relatam que as doses de 1,5 e 2,0 mg i.a./vaso resultaram em plantas excessivamente pequenas, enquanto doses menores $(0,5$ e 1,0 mg i.a./vaso) forneceram plantas mais harmoniosas, com alturas adequadas ao mercado.

SACHS et al. (1976) sugere que a altura de plantas cultivadas em vaso deve ser de 1,5 a 2 vezes a altura do vaso. Para o tamanho de vaso utilizado neste experimento a faixa adequada de altura de planta situa-se entre $18 \mathrm{e}$ $24 \mathrm{~cm}$, sendo a dose de $6 \mathrm{mg}$ i.a/vaso a que proporcionou 


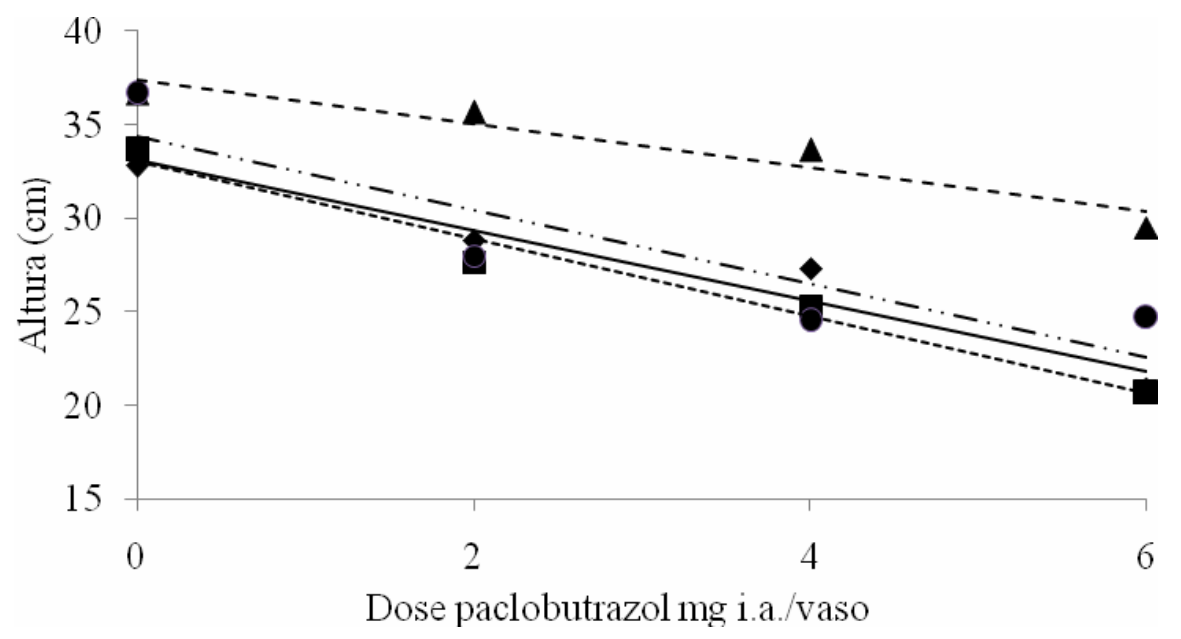

$\begin{array}{ll}\text { Substrato 1: } \mathrm{Y}=33,08-1,875 \mathrm{x} & \mathrm{R}^{2}=0,94 \\ \text { Substrato 2: } \mathrm{Y}=33,03-2,067 \mathrm{x} & \mathrm{R}^{2}=0,97 \\ \text { Substrato 3: } \mathrm{Y}=37,04-1,175 \mathrm{x} & \mathrm{R}^{2}=0,92 \\ \text { Substrato 4: } \mathrm{Y}=34,38-1,975 \mathrm{x} & \mathrm{R}^{2}=0,80\end{array}$

Figura1. Altura de plantas de girassol (var. Golden), plantadas em diferentes substratos, submetidas a doses de paclobutrazol. Figure 1. Sunflower plant height (Golden cultivar), grown in different substrates, submitted to paclobutrazol dosages.

altura ideal de plantas de girassol $(23,9 \mathrm{~cm})$.

Com relação ao número de folhas, a análise da variância mostrou efeito do substrato, constatando-se maior número de folhas, em média, quando as plantas de girassol foram cultivadas no $\mathrm{S} 1$, resultado, estatisticamente, superior aos obtidos com o S4 e igual aos demais tratamentos (tabela 1). De acordo com as exigências do mercado, plantas envasadas devem ter porte compacto e grande número de folhas para uma melhor harmonia do vaso.

Tabela 1. Valores médios para número de folhas de girassol em diferentes substratos Table 1. Leaf number of sunflower plants grown in different substrates.

\begin{tabular}{lc}
\hline \multicolumn{1}{c}{ Substrato } & Média \\
\hline S1: solo:areia:casca de arroz carbonizada, $(2: 0,5: 2 ; \mathrm{v} / \mathrm{v})$ & $17,08 \mathrm{~A}$ \\
S2: solo:areia:carvão de cana $(2: 0,5: 2 \mathrm{v} / \mathrm{v})$ & $16,25 \mathrm{AB}$ \\
S3: solo:areia:carvão vegetal $(2: 0,5: 2 \mathrm{v} / \mathrm{v})$ & $15,41 \mathrm{AB}$ \\
S4: substrato comercial & $14,50 \mathrm{~B}$ \\
\hline
\end{tabular}

Médias seguidas pela mesma letra não diferem entre si ao nível de $5 \%$ pelo teste de Tukey.

A análise da variância da concentração de clorofila obtida com o auxílio de um medidor demonstrou efeito de doses de paclobutrazol, e as unidades SPAD seguiram tendência quadrática em função das doses. Obteve-se o ponto de máxima concentração de clorofila na dose de $5,08 \mathrm{mg}$ i.a./vaso, equivalente a 27,18 unidades SPAD (Figura 2). A maior concentração de clorofila nas folhas intensificou a sua coloração verde, o que está relacionado com a redução da expansão das células pelo uso do retardante de crescimento (MÉTRAUX, 1987). AURAS (1997) também encontrou aumento na concentração de clorofila em plantas de girassol cultivar GR16, cultivado como oleaginosa, quando submetidas a doses de paclobutrazol. Esta maior intensificação da cor verde das folhas pelo uso do paclobutrazol possibilita maior contraste entre o verde das folhas e o amarelo da inflorescência; há aumento da atratividade visual e, desse modo, favorecimento à comercialização. 


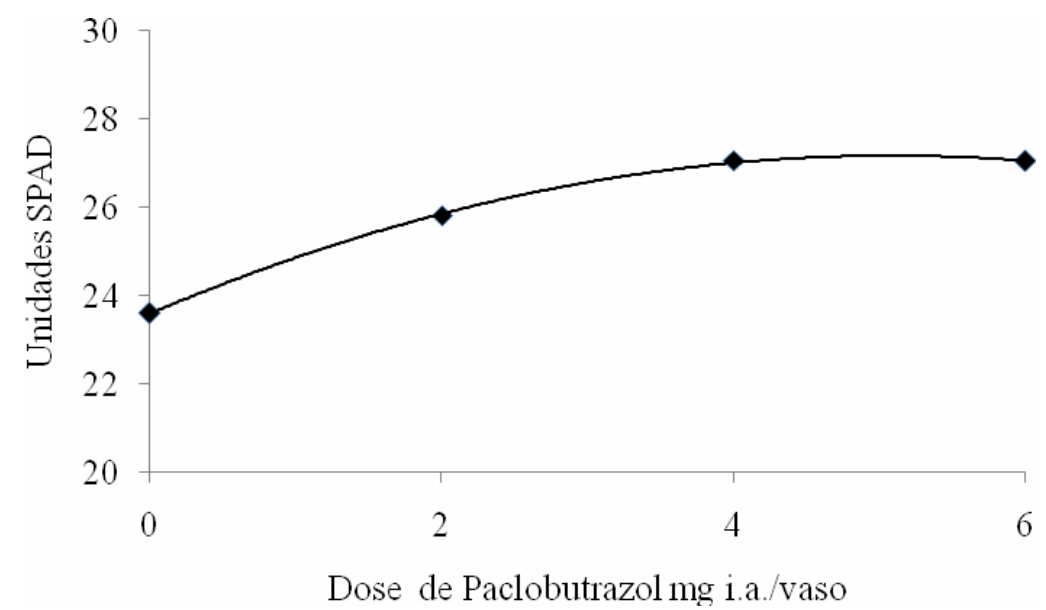

Figura 2. Comportamento do nível SPAD em resposta a doses de paclobutrazol.

Figure 2. Leaf SPAD level in response to paclobutrazol dosages.

\section{CONCLUSÕES}

Os substratos S1 (solo, areia, casca de arroz carbonizada, 2:0,5:2) e S2 (solo, areia, carvão vegetal, 2:0,5:2) foram mais eficientes na redução da altura das plantas de girassol. A aplicação de paclobutrazol foi eficiente em reduzir a altura das plantas de girassol, sem causar sintomas de toxidez.

A dose de $6 \mathrm{mg}$ i.a./vaso possibilitou redução satisfatória do porte das plantas, conferindo a harmonia de vaso desejável, sendo a mais indicada, exceto para o substrato 3 (solo:areia:carvão vegetal).

\section{REFERÊNCIAS}

ALMEIDA, J.A.S. de; PEREIRA, M. de F.D.A. Efeito de $\mathrm{GA}_{3}$ e Paclobutrazol no desenvolvimento vegetativo do girassol. Revista Brasileira de Fisiologia Vegetal, Campinas SP. v.9, n.1, p.55-60, 1996.

AURAS, N.E. Efeitos do paclobutrazol sobre morfologia e anatomia foliar, crescimento de parte aérea, distribuição de biomassa e trocas gasosas em girassol. Viçosa: UFV, 1997. 88p. Tese (Doutorado em Fisiologia Vegetal) - UFV.

BARBOSA, J.G. Crisântemos - Produção de mudas; Cultivo para corte de flor; Cultivo em vaso e Cultivo hidropônico. Viçosa: Aprenda Fácil Editora, 2003. 220p. DASOJU, S., EVANS, M.R., WHIPKER, B.E. Paclobutrazol drenches control growth of potted sunflower. HortTechnology, New York, v.8(2):235-237, 1998.

HUNTER, D.M.; PROCTOR, J.T.A. Paclobutrazol affects growth and fruit composition of potted grapevines. HortScience, Alexandria, v.27, n.4, p.319-321, 1992.

MAUK, C.S.; UNRATH, C.R.; BLANKENSHIP, S.M.; LEHMAN, L.J. Influence of method of application of paclobutrazol on soil residues and growth retardation in a "STARKRINSON - Delicious" apple orchand. Plant Growth Regulation, Heidelberg, v.9, p.27-35, 1990.

MÉTRAUX, J.P. Gibberellins and plant cell elongation. In: DAVIES, P.J. ed. Plant hormones and their role in plant growth and development. Dordrecht, Martinus Nijhoff Publishers, 1987. p.296 317.

NORCINI, J.G., HUDSON, W.G., GARBER, M.P., JONES, R.K., CHASE, A.R., BONDARI, K. Pest management in the U. S. greenhouse and nursey industry: III. Plant growth regulation. HortTechnology, New York, v.6, n.3, p.207-210, 1996.

PENG, L.Z.; HE, S.L.; TANG, J.; XU, E.; SONG, N.J. Absorption and translocation of $3 \mathrm{H}$-labeled paclolbutrazol in citrus. Acta Horticulturae, Vertemate com Minoprio, v.21, p.7-12, 1994.

SACHS, R.M.; KOFRANEK, A.M.; HACKETT, W.P. Evaluation new pot plant species. Florist's Rev. Topeka, 159(4116):80-84, 1976.

SETIA, R.C.; BRATHAL, G; SETIA, N. Influence of paclobutrazol on growth and yield of Brassica Carinata ABr. Plant Growth Regulation, Heidelberg, v.126, p.121127, 1995.

TINOCO, S.A.; MUNIZ, M.A.; BARBOSA, M.S.; RODRIGUES, H.P.; GROSSI, J.A.S.; BARBOSA, J.G. Qualidade de plantas de crisântemo de vaso tratadas com paclobutrazol. In: Fórum Latino-Americano de Plantas Ornamentais, 2004, Nova Petrópolis. Fórum LatinoAmericano de Plantas Ornamentais. Porto Alegre: AFLORI, 2004. p.134-136.

WEAVER, J.R. Reguladores del crecimiento en la agricultura. Primera edición. Editorial Trillas. México, D.F., 1989. 141p. 